\begin{tabular}{|c|c|}
\hline Title & $\begin{array}{l}\text { Microbial diversity in the human intestine and novel insights from } \\
\text { metagenomics }\end{array}$ \\
\hline Authors & $\begin{array}{l}\text { Ventura, Marco;Turroni, Francesca;Canchaya, Carlos;Vaughan, } \\
\text { Elaine E.;O'Toole, Paul W.;van Sinderen, Douwe }\end{array}$ \\
\hline Publication date & 2009-01-01 \\
\hline Original Citation & $\begin{array}{l}\text { Ventura, M, Turroni, F, Canchaya, C, Vaughan, EE, O'Toole, PW, van } \\
\text { Sinderen, D (2009) 'Microbial Diversity In The Human Intestine and } \\
\text { Novel Insights From Metagenomics'. Frontiers In Bioscience, } 14 \\
\text { (1):3214-3863. }\end{array}$ \\
\hline Type of publication & Article (peer-reviewed) \\
\hline $\begin{array}{l}\text { Link to publisher's } \\
\text { version }\end{array}$ & $10.2741 / 3445$ \\
\hline Rights & (C) Frontiers in Bioscience \\
\hline Download date & 2023-04-26 12:16:32 \\
\hline $\begin{array}{l}\text { Item downloaded } \\
\text { from }\end{array}$ & http://hdl.handle.net/10468/117 \\
\hline
\end{tabular}

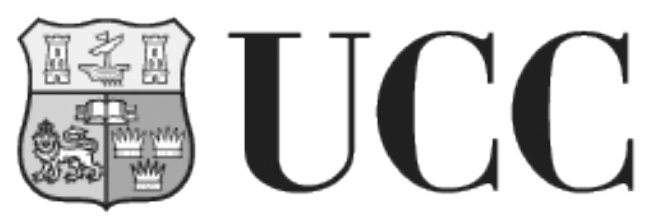

University College Cork, Ireland Coláiste na hOllscoile Corcaigh 


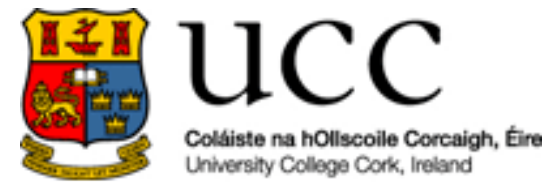

\author{
Ventura M, Turroni F, Canchaya C, Vaughan EE, O'Toole PW, van \\ Sinderen D. (2009) Microbial diversity in the human intestine and \\ novel insights from metagenomics. Frontiers in Bioscience, 14 (1) \\ pp. 3214-3221
}

"This is an, un-copyedited, author manuscript that has been accepted for publication in the Frontiers in Bioscience". Cite this article as appearing in the Journal of Frontiers in Bioscience. Full citation can be found by searching the Frontiers in Bioscience (http://bioscience.org/search/authors/htm/search.htm) following publication and at PubMed (http://www.ncbi.nlm.nih.gov/entrez/query.fcgi?CMD=search\&DB=pubmed) following indexing.

This article may not be duplicated or reproduced, other than for personal use or within the rule of "Fair Use of Copyrighted Materials" (section 107, Title 17, U.S. Code) without permission of the copyright holder, the Frontiers in Bioscience. From the time of acceptance following peer review, the full final copy edited article of this manuscript will be made available at http://www.bioscience.org/. The Frontiers in Bioscience disclaims any responsibility or liability for errors or omissions in this version of the un-copyedited manuscript or in any version derived from it by the National Institutes of Health or other parties."

Deposited on: 15 February 2010 


\title{
Microbial diversity in the human intestine and novel insights from metagenomics
}

\author{
Marco Ventura ${ }^{1}$, Francesca Turroni ${ }^{1}$, Carlos Canchaya ${ }^{1}$, Elaine E. Vaughan ${ }^{2}$, Paul W. O’Toole ${ }^{3}$, Douwe van Sinderen ${ }^{3}$ \\ ${ }^{1}$ Department of Genetics, Biology of Microorganisms, Anthropology and Evolution, University of Parma, Italy, ${ }^{2}$ Unilever Food \\ and Health Research Institute (UFHRI), ${ }^{3}$ Alimentary Pharmabiotic Centre and Department of Microbiology, University College \\ Cork, Western Road, Cork, Ireland
}

\section{TABLE OF CONTENTS}

\section{Abstract}

\section{Introduction}

3. Microbiota diversity of the human git as determined by molecular methods based on $16 S$ rRNA

4. Factors influencing the composition of the gastro intestinal microbiota

5. Development of the human intestinal microbiota

6. Microbial diversity assessed by compositional metagenomics

6.1. Advantages and disadvantages of $16 S$ rRNA- and metagenomic clone library-based diversity approaches

7. Conclusions

8. Acknowledgments

9. Reference

\section{ABSTRACT}

Bacterial communities reside in very different ecological niches on and within the human host, such as those associated with the alimentary tract. The human gastrointestinal tract is populated with as many as 100 trillion bacterial cells, whose collective genome likely reflects the co-evolution between the microbial community and its host. Recent progress has highlighted the intriguing diversity of these bacterial populations and their important contributions to human physiology. Thus, a thorough understanding of the autochthonous component of the intestinal microbiota is expected to provide crucial information not only on how to develop therapies for various gastrointestinal diseases but also on how to choose the next generation of probiotic bacteria as part of novel functional foods. Recently, novel culture-independent approaches such as metagenomics-based techniques were shown to be crucially important for the exploration of the biodiversity of the human intestinal microbiota.

\section{INTRODUCTION}

Mammals, including humans, have, throughout their evolution, been associated with complex microbial communities, which inhabit the surfaces and alimentary tract of their host and which outnumber the human somatic and germ cells by a factor of ten (1). The distal part of the gut, i.e. the intestine, represents the environment where microbial diversity is high while its abundance is at its theoretical maximum. The human intestinal microbiota is estimated to be composed of $10^{13}$ to $10^{14}$ microorganisms whose collective genome, the microbiome, contains at least 100-fold more genes than the complete human genome (2). This complex microbiota is considered to be critically important for various gut functions, such as host nutrition, regulation of epithelial development (3), regulation of host fat storage (4), stimulation of intestinal angiogenesis (5), inflammatory immune responses $(6$.$) and pathogen resistance (7,8)$.

Deviations in the composition of the microbiota from the typical adult pattern as well as from the canonical infant pattern have been associated with a variety of conditions ranging from inflammatory bowel disease to allergy, obesity and atopic disease (9). Thus, our desire to understand intestinal microbial ecology is linked to our wish to manage and maintain human health. The composition of the adult intestinal microbiota has been intensively studied, by means of culture-based methodologies and, more recently, through culture-independent technologies based on the amplification and direct sequencing of small subunit ribosomal DNA sequences $(10,11,12,13)$. The intestinal microbiota have also been analysed by other, recently developed community profiling culture-independent techniques, including DNA-microarray, fluorescent in situ hybridization (FISH), and methods such as PCR coupled to denaturing gradient and temperature electrophoresis (PCR-DGGE and PCR-TGGE) (for review see 14). The microbial populations that reside in the distal human intestine are predicted to consist of more than 400 bacterial species and around 1,000 phylotypes, belonging to a limited number of broad taxonomic units $(11,13)$. Within these units, members of the anaerobic genera such as Bacteroides, Eubacterium, Bifidobacterium, Ruminococcus, Clostridium and Faecalibacterium have been found to constitute the majority of the adult human intestinal microbiota, representing a relatively constant bacterial community that remains relatively stable over time $(11,14)$. Conversely, the infant intestinal microbiota appears to be much more simple and variable in its composition, and less stable over time (15). 
In this review we will focus on the current knowledge regarding diversity and development of the human gut microbiota as determined by molecular $16 \mathrm{~S}$ ribosomal RNA approaches, as well as on the various identified factors that govern its establishment. We further discuss metagenomics approaches as a powerful tool to explore the biodiversity of the intestinal microbiota.

\section{MICROBIOTA DIVERSITY OF THE HUMAN GIT AS DETERMINED BY MOLECULAR METHODS BASED ON 16S rRNA}

The human gut ecosystem is very dynamic and consists of various bacterial populations that are either permanent gut residents, i.e. autochthonous components, or transient inhabitants, i.e. allochthonous members, introduced from the environment. The relationship between bacteria and the human host can be viewed as a continuum between symbiosis, commensalism and pathogenesis, where the former two relationships can be considered as mutualistic. The human intestine is assumed to be colonized by a vast array of symbionts and commensals that provide various metabolic capabilities, which humans consequently did not need to develop themselves (16). The genetic diversity found in the human intestine is believed to be the result of intensive selection and co-evolution, where Horizontal Gene Transfer (HGT) has been suggested to act as the principal evolutionary force in shaping the human microbiome (15).

The human intestinal microbiota is characterized by a relatively small number of bacterial and archaeal divisions, compared to other environments such as soil or stromatolite (17). Culture-independent approaches based on 16S rRNA gene sequences revealed that the intestinal human microbiota typically comprises representatives of only nine major bacterial divisions, i.e., Bacteroidetes, Firmicutes, Fusobacteria, Proteobacteria, Verrucomicrobia, Cyanobacteria, Spirochaeates, VadinBE97 and Actinobacteria $(13,15)$. Notably, proportions and compositions of Bacteroidetes and bifidobacteria were consistently stable within individuals whereas the Clostridium group (e.g. Clostridium coccoides and Clostridium leptum) showed large inter-individual variation (18). Thus, the microbial composition of such ecosystems is relatively simple compared to other habitats such as the soil environment, which contains at least 20 bacterial divisions (17). With respect to the phylogenetic architecture of the human intestinal microbiota, it has been suggested that this is derived from the diversification of a discrete, and therefore restricted initial microbial community. Notably, the limited diversification of the intestinal microbiota may reflect the relatively short time of existence of the human intestine as a habitat, i.e. 100 million years for mammals, as opposed to over 3.85 billion years for the ocean (19).

\section{FACTORS INFLUENCING THE COMPOSITION OF THE GASTRO INTESTINAL MICROBIOTA}

The microbiota of the adult GIT is composed of microorganisms that represent superior competitors for a fixed set of niches (15). This interpretation prompted an intensive search for factors that define a microbial intestinal niche such as the genotype of the host and the diet. However, human microbial communities are also influenced by other, less predictable factors such as interactions between individual resident members of the microbiota, as well as transient members such as pathogens and probiotics.

The genotype of the host is thought to partly explain variation of the GIT microbiota between individuals through the differential action of the genes that specify and control the immune system (20). For example, the immune system of the host influences the microbiota composition by restricting microbial penetration in the host tissues $(21,22)$. Furthermore, the genotype of the host modulates the intestinal microbiota through the availability of specific attachment sites and host-derived resources. In the case of Bacteroides thetaiotaomicron, which is considered the model commensal enteric microorganism, it has been shown that this bacterium regulates the production of fucosylated glycans by the host epithelium in order to respond to the bacterial utilization of fucose as a growth substrate (1). Although such interactions have been studied in murine models, it is plausible that they also occur in humans (1). If this is indeed the case, they may be influenced by human polymorphisms in fucosyltransferase enzymes, which are responsible for different blood types (23).

The effect of genotype on the composition of the human microbiota was demonstrated in a gut ecological study involving monozygotic twins (24). According to this report, there were greater similarities between the gut microbiota of monozygotic twins than between monozygotic twins and their unrelated marital partners. The monozygotic twins still exhibited small differences in their intestinal microbiota, suggesting that although host genotype does appear to influence the intestinal microbiota, genetically identical subjects living in very similar environments can still exhibit significant differences in their intestinal microbiota (24). The importance of the initial colonizing microbial community on the eventual microbial composition of the gut is evident from animal studies. The composition of the mouse gut microbiota is significantly affected by maternal transmission (25). Similarly, investigations in which the microbiota was transferred from one host to another, from normal mice or zebrafish to axenic mice or zebrafish, showed that the microbial community which establishes itself in the gut at the time of birth, together with the features of the gut habitat, are determining factors for the microbiota composition (26).

Diet is also a pivotal variable in influencing the composition of the intestinal microbiota. Studies on chemically well defined diet components have proven a clear correlation between diet and the presence of specific bacterial groups. It has been 
shown that a diet rich in inulin and related fibers promote an increase in bifidobacteria (27), whereas the intake of dietary sulphate favours several genera of sulphate-reducing bacteria over methanogenic Archaea (28). Diet affects not only the microbiota composition, but more significantly the metabolic activities of the microorganisms $(29,30)$. These are also influenced by factors relating to the gut environment, i.e. local conditions of $\mathrm{pH}$, oxygen and hydrogen, metabolite concentration and gut transit time (31). However, representative strains of several bacterial genera were shown to be generalists with comparable growth abilities on overlapping ranges of substrates $(27,28)$. In addition, it should be noted that mucin and other host-derived compounds are on their own already sufficient to support a diverse microbial community $(32,33)$. Bacterial interactions together with resource competition are considered crucial determinants of intestinal niches (34). Bacterial co-operations in the GIT happen during the breakdown of complex carbohydrates, which originate from the diet or host (35).

Finally, bacterial diversity in the human GIT might be influenced by the action of antimicrobial compounds such as bacteriocins or bacteriophages, which can promote complex dynamics due to evolutionary trade-offs between resistance and sensitivity (36), and which can also provide protection against bacterial infections (8). Recently, a comparative analysis was performed between healthy individuals and patients with Crohn's disease (CD) with respect to their gut mucosa-associated bacteriophage community. Based on the results, it was postulated that bacteriophages play a key role in the dysregulated immune response of IBD patients to the mucosal-associated bacterial population (38).

\section{DEVELOPMENT OF THE HUMAN INTESTINAL MICROBIOTA}

Following birth every human (baby) develops a complex and active intestinal ecosystem starting from an assumed sterile environment. In just a matter of a couple of days the GIT of a newborn becomes home to a large bacterial community, whose total number quickly exceeds the total number of cells of its host (15). However, there are several controversial reports regarding the composition of the infant GIT microbiota and about the factors that influence it $(15,38)$. PCR based techniques such as PCR-DGGE revealed that species most closely related to the genera Bifidobacterium and Ruminococcus dominate the intestinal microbiota of infants during the first year of life (39). The GIT microbiota of infants delivered by caesarean section has been described to differ significantly from that of infants delivered vaginally $(15,38)$, which would be consistent with the finding that the infant GIT microbiota harbours elements of the maternal vaginal microbiota (40). Furthermore, the gestational outcome, for example hospitalized preterm infants versus healthy full-term babies, dramatically influences the composition of the developing GIT microbiota $(41,42)$. This could be influenced by the immune system maturity and age of priming.

Considering the importance of the symbiosis between intestinal microbes and the human host, it is plausible that the development of the microbiota in the infant intestine would have evolved under strong selective pressure, acting on both the intestinal niche as well as on its bacterial colonizers. So a restricted number of co-evolved commensal bacteria may be exceptionally well adapted to occupy the intestinal ecological niche of the infant intestine. It has been found that the GIT microbiota in the first month of life is highly variable between individuals. The intestinal microbiota that first develops immediately following delivery is to a significant extent influenced by specific bacteria to which the infant is exposed in its environment. Such environmental exposures are likely to include elements of the maternal vaginal, fecal, and skin microbiota (15). Over time, however, the ecological fitness advantages of specific bacterial groups that typically dominate the adult colonic microbiota lead to a displacement or shift in numbers of the initial bacterial colonizers. In addition, progressive changes in the GIT environment, due to intrinsic developmental modifications of the GIT mucosa as well as transition to a more "adult" diet, combined with effects elicited by the microbiota themselves may provide the selection for the most highly adapted bacteria (43).

\section{MICROBIAL DIVERSITY ASSESSED BY COMPOSITIONAL METAGENOMICS}

Over the last decade, many investigations have focused on culture-independent approaches to evaluate the complexity of the intestinal microbiota. Among these novel investigative methods that are used to study the microbial ecology of complex bacterial communities, the so-called metagenomics approach is considered to represent the method that produces results of 'gold standard' quality. Metagenomics is the study of microbial communities through sequence-based, compositional and/or functional analyses of all the combined microbial genomes contained within an environmental sample (Figure 1). With respect to its application to human intestinal microbiota, metagenomic studies of mucosal as well as faecal samples revealed the presence of representatives of the phylogenetic groups Firmicutes, Bacteroidetes, Proteobacteria, Fusobacteria, Verrucomicrobia and Actinobacteria $(11,12)$. However, a large portion of the phylotypes detected belong to the Bacteroidetes and Firmicutes phyla, while only one of the detected species belonging to the methanogenic archaeon, Methanobrevibacterium smithii $(11,12,44,45)$. Notably, with respect to phylotypic variation among the microbiota, it was found that only the Firmicutes phylum was comprised of a large number of phylogenetic types (e.g., the clostridial class). Also, an elevated number of phylotypes are related to butyrate-producing bacteria, all of which fall within the clostridial clusters IV, XIVa and XVI of Collins et al. (46). The other most common bacterial phylum detected in human gut samples is the Bacteroidetes with a small number of representative species, frequently including members of the Bacteroides fragilis subgroup, Bacteroides uniformis, Bacteroides thetaiotaomicron and Bacteroides distasonis (12). Recently, a compositional metagenomic study involving healthy individuals as well as with Crohn's disease revealed a reduced complexity of the bacterial phylum Firmicutes as well as over-representation of species such as uncultured Porphyromonadaceae as a signature of the faecal microbiota in patients with CD (47). 
The bacterial phyla appear to be unevenly distributed along the length of GIT. Metagenomic analyses of jejunum samples revealed a distinctive community composition which included an abundance of bacteria belonging to the Streptococcus genus, whereas Bacteroidetes and Clostridium genera were the predominant microbial groups identified in the distinctive composition of the microbiota in the distal ileum, ascending colon and rectum (12).

Notably, bacterial diversity within the human intestine and faeces is very high while also displaying a significant intersubject variability (11). It has been suggested that the faecal microbiota represents a combination of shed mucosal bacteria (autochthonous components of the intestinal microbiota) and a separate non-adherent luminal population (allochthonous components of the intestinal microbiota) (48-49).

\subsection{Advantages and disadvantages of 16S rRNA- and metagenomic clone library-based diversity approaches}

The scientific rigor of metagenomic technology in defining the bacterial composition of complex bacterial communities is widely accepted (14) (Table 1). This method clearly emphasizes the impact of genomics on microbial ecology (50) and combines the use of molecular biology and genetics to identify and characterise genetic material from complex environments (51). In contrast to culture-dependent approaches, metagenomic investigations can overcome many technical limitations and problems that are linked to the handling of exigent bacteria such as those from intestinal environments. Moreover the combination of PCR of $16 \mathrm{~S}$ rRNA gene and direct sequencing has expanded our knowledge of microbial diversity; it has been shown that less than $1 \%$ of the microbial communities in soil are accessible through culture-based techniques, and it is likely that this situation is very similar for many other microbial niches including the GIT. In addition, the proportion of non-culturable bacteria appears to increase with the age of the human subject, because the number of novel phylotypes was strikingly high when the microbiota of elderly subjects was analyzed by $16 \mathrm{~S}$ clone library sequencing and T-RFLP (52). This highlights the importance of a culture-independent method for assessing the microbial GIT community structure in these individuals.

Metagenomic analysis has significant advantages. For example it allows a high throughput approach to process samples, which contrasts significantly with the time-consuming and labour-intensive classical culturing techniques. Moreover, metagenomic studies do not require immediate processing, as samples can be frozen and subjected to analysis later, while DNA can easily be transported between laboratories (51). However, the metagenomic approach is not free from any drawbacks. Indeed, there are currently few studies on diversity from metagenomic clone libraries but it is expected that as high through put sequencing becomes cheaper, metagenomics as a routine quantitative method for diversity analysis will be within the reach of most laboratories. It is rather complicated with respect to the standardization of extraction of genetic material to cover every member of a given bacterial community. In fact, the results achieved by metagenomics studies may be significantly influenced by the efficacy of the protocols used for directly extracting DNA from environmental samples (e.g., fecal or human tissues) as well as by the specificity of PCR primers and associated PCR conditions (53). Also for Proteobacteria, Fusobacteria and Verrucomicrobia phyla some contradicting results are described $(11,12)$. A relatively small number of sequences corresponding to the above mentioned taxa were found in one particular metagenomic study (11), in contrast to another investigation of human intestinal biopsies which revealed a large number and proportion of these phylotypes. In addition to the bacterial phyla describe above there may be other groups (e.g. Actinobacteria) for which some methodological problems may be present. For instance, very often bifidobacteria have not detected $(10,14)$ or have been underestimated $(11)$ by metagenomic approaches, which may be related to low amplification efficiencies due to high $\mathrm{G}+\mathrm{C}$ content. Furthermore, even if metagenomic investigations of human intestinal microbiota generate very valuable data that outline the microbial diversity of this niche, they should nevertheless be interpreted with caution not only because of technical limitations outlined above, but also because the large majority of published metagenomic data are based on faecal samples rather than intestinal biopsies. Thus, the identified phylotypes do not necessarily represent the actual diversity of the intestinal microbiota (54). In fact, the faecal microbial community (or at least the microbial DNA present in faeces) represents all bacterial species living in the GIT and so metagenomic analysis of faecal samples seems to overestimate the diversity of the human gut microbiota, a notion which was corroborated by the finding that the microbiota of the proximal regions of the human GIT is different from that present in other parts of the intestine (55). Despite this, faecal samples are often chosen to investigate the microbial composition of the intestinal microflora because from a practical, clinical and ethical perspective they are easy to collect. A further limitation of the available metagenomic data is that the composition of the predominant bacterial community is host-specific (24) and that the number of genes within a given community is so large that the number of analyzed fragments derived from a single sample must be enormous to obtain a statistically relevant representation of the complete microbiome (56). Furthermore, not all elements of a library, even if such a library were to be complete, are sequenced and bacterial species that are only minor components of the microbiota may appear to be absent. Therefore, considerable caution must be applied in the design, analysis and interpretation of the results obtained by metagenomic studies (13). Finally, there are sometimes difficulties in cloning DNA from Gram positive bacteria into the commonly used laboratory Gram negative bacterial hosts.

\section{CONCLUSIONS}

Despite the presumed importance played by the GIT microbiota in maintaining a healthy human host, large knowledge gaps still exist with respect to the microbial composition, its development and strain diversity, as well as their associated metabolic effects. In fact, diversity (at the 16S rRNA level) is currently a biomarker for functional activity of specific microbes and ultimately for health effect. Recently, culture-independent studies, in particular metagenomic investigations, have significantly expanded our knowledge of the human intestinal microbiota. It has been shown that the composition of gut 
communities is variable over time with certain bacterial components acting as superior competitors for all available niches. Moreover, functional analyses of the intestinal microbiota, through sequence exploration of metagenomic libraries or by means of micro arrays that were based on key intestinal commensal bacteria, have provided in depth information as to how these bacteria function within their host and how they interact with that host and with each other. Novel sequencing technologies (e.g. pyrosequencing techniques) coupled with development of bioinformatic means will provide more in depth analysis of the biodiversity existing in the intestinal microbiota. Moreover, the expansion of high through put functional genomic tools, e.g. micro array technologies will provide the necessary postgenomic platforms to investigate the functionality of human bacterial genomes in the human gut. Moreover, other metagenomics approaches such as the total microbiota genome sequencing might be important in order to identify new phylotypes with no previous cultured relatives. The analysis of such genome sequences together with functional genomics approaches will be crucial in order to highlight sequence redundancy and thus may provide very valuable information on both, strategies for survival under GIT conditions and processes behind the mutualistic relationship established between certain microorganisms and the host. In fact, symbionts may dedicate part of their genomes to functions that are beneficial for both bacterium and host. Thus, the identification of such functions will aid in the development of novel probiotics.

Novel "omics" technologies such as transcriptomics, proteomics and metabonomics, will open new avenues of research in the study of the functionality of the human gut microbiota and may explain the role played by certain microbiota's molecules (e.g., metabolites) in the developing of diseaseor in the establishment of health.

Understanding the composition of the intestinal microbiota represents a crucial step towards a knowledge-based design of functional foods or for the selection of novel probiotic bacteria. For example, it is important that any newly developed probiotic bacterium must be genetically adapted to survive in the intestinal ecological niche. Such requirements will provide these bacteria with genetically determined capabilities to interact with both the human host as well as with the other indigenous components of the intestinal microflora, thus allowing them to exert their health-promoting effect(s) (e.g., probiotic role). Moreover, probiotic bacteria genetically adapted to live in the human intestinal niche will likely encode various "biological defences" to counteract the establishment of potentially dangerous microorganisms. Greater depth of knowledge on the autochthonous component of the intestinal microbiota will provide the possibility to screen or develop important characteristics within novel probiotic microbes, including the capacity to be highly stable in the human intestine and especially have reliable or enhanced probiotic effects. Furthermore as our understanding of the ecology and rich diversity of the human gut microbiota develops, members isolated from intestinal biopsies (autochthonous component of the intestinal microbiota), beside faecal isolates, will contribute to the next generation of probiotic microbes. Furthermore, the exploration of human gut microbiota will possibly lead in the long term to the identification of novel probiotics, that are different from the commonly used lactobacilli and bifidobacteria and that possess new health benefits. In addition, other criteria that should be taken into account when selecting novel probiotics are the differentiation of strains targeted for different host groups such as adult versus children or elderly people, and those that are compatible with the genetic background of the targeted host (57). Greater knowledge on evolution of species and strain diversity in the intestine can thus open up new possibilities that are expected to lead to the development of a next generation of designer probiotics with clearly described and scientifically supported health benefits.

\section{ACKNOWLEDGMENTS}

This work was financially supported by Science Foundation Ireland through the Alimentary Pharmabiotic Centre (POT, DvS), by the Dept. Agriculture and Food FHRI award to the ELDERMET consortium (POT, DvS), by the Italian Award for Outstanding Young Researcher scheme "Incentivazione alla mobilita' di studiosi stranieri e italiani residenti all'estero" to the Marie Curie Reintegration Grant (MERG-CT-2005-03080) and by Parmalat spa, Italy to MV.

\section{RFERENCE}

1. L. V. Hooper, J. Xu, P. G. Falk, T. Midtvedt and J. I. Gordon: A molecular sensor that allows a gut commensal to control its nutrient foundation in a competitive ecosystem. Proc Natl Acad Sci 96, 9833-9838 (1999)

2. S. R. Gill, M. Pop, R. T. Deboy and other authors: Metagenomic analysis of the human distal gut microbiome. Science 312 , 1355-1359 (2006)

3. S. Rakoff-Nahoum, J. Paglino, F. Eslami-Varzaneh, S. Edberg and R. Medzhitov: Recognition of commensal microfolora by toll-like receptors is required for intestinal homeostasis. Cell 118, 229-241 (2004)

4. F. Backhed, H. Ding, T. Wang, L. V. Hooper, G. Y. Koh, A. Nagy, C. F. Semenkovich, and J. I. Gordon: The gut microbiota as an environmental factor that regulates fat storage. Proc Natl Acad Sci U S A 101, 15718-15723 (2004)

5. T. S. Stappenbeck, L. V. Hooper, J. I. Gordon: Developmental regulation of intestinal angiogenesis by indigenous microbes via Paneth cells. Proc Natl Acad Sci 99:15451-15455 (2002) 
6. M. C. Noverr and G. B. Huffnagle: Does the microbiota regulate immune responses outside the gut? Trends Microbiol. 12, $562-568(2004)$

7. D. C. Savage: Microbial ecology of the gastrointestinal tract. Annu. Rev. Microbiol. 31, 107-133 (1977)

8. S.C. Corr, Y. Li, C.U. Riedel, P.W. O'Toole, C. Hill, and C.G. Gahan. Bacteriocin production as a mechanism for the antiinfective activity of Lactobacillus salivarius UCC118. Proc Natl Acad Sci U S A. 2007 104:7617-21 (2007)

9. R. E. Ley, D. A. Peterson and J. I. Gordon: Ecological and evolutionary forces shaping microbial diversity in the human intestine. Cell 124, 837-848 (2006)

10. E. E. Vaughan, F. Shut, H. G. Heilig, E. G. Zoetendal and V. W. deVos: A molecular view of the intestinal ecosystem. Curr. Issues Intest. Microbiol. 1, 1-12 (2000)

11. P. B. Eckburg, E. M. Bik, C. N. Bernstein, E. Purdom, L. Dethlefsen, M. Sargent, S. R. Gill, K. E. Nelson and D. A. Relman: Diversity of the human intestinal microbial flora. Science 308, 1635-1638 (2005)

12. M. Wang, S. Ahrne, B. Jeppsson and G. Molin: Comparison of bacterial diversity along the human intestinal tract by direct cloning and sequencing of 16S rRNA genes. FEMS Microbiol Ecol 54, 219-231 (2005)

13. M. Rajilić-Stojanović, H. Smidt, \& W.M. de Vos. Diversity of the human gastrointestinal tract microbiota revisited. Environ Microbiol. 9:2125-36 (2007)

14. F. Turroni, A. Ribbera, E. Foroni, D. van Sinderen, and M. Ventura. Human gut microbiota and bifidobacteria: from composition to functionality. Antonie van Leeuwenhoek (2008)

15. C. Palmer, E. M. Bik, D. B. Digiulio, D. A. Relman and P. O. Brown: Development of the Human Infant Intestinal Microbiota. PLoS Biol 5, e177 (2007)

16. D.C. Savage. Microbial biota of the human intestine: a tribute to some pioneering scientists. Curr Issues Intest Microbiol. 2 , $1-15(2001)$

17. J. Dunbar, S. M. Barns, L. O.Ticknor and C. R. Kuske: Empirical and theoretical bacterial diversity in four Arizona soils. Appl Environ Microbiol 68: 3035-45 (2002)

18. C. Lay, L. Rigottier-Gois, K. Holmstrom, M. Rajilic, E.E. Vaughan, W.M. de Vos, M.D. Collin, R. Thiel, P. Namsolleck, M. Blaut and J. Dore. Colonic microbiota signatures across five northern European countries. Appl. Environ. Microbiol. 71, 4153$4155(2005)$

19. W.J. Murphy, E. Eizirik, S.J. O’Brien, O. Madsen, M. Scally, C.J. Douady, E. Teeling, O.A. Ryder, M.J. Stanhope, W.W. de Jong and M.S. Springer. Resolution of the early placental mammal radiation using Bayesian phylogenetics. Science 294, 2348$2351(2001)$

20. R. E. Ley, P. J. Turnbaugh, S. Klein and J. I. Gordon: Microbial ecology: human gut microbes associated with obesity. Nature 444, 1022-1023 (2006)

21. B. A. Cobb, Q. Wang, A. O. Tzianabos and D. L. Kasper: Polysaccharide processing and presentation by the MHCII pathway. Cell 117, 677-687 (2004)

22. T. T. Macdonald and G. Monteleone: Immunity, inflammation, and allergy in the gut. Science 307, 1920-1925 (2005)

23. D.J. Becker and J.B. Lowe. Fucose: biosynthesis and biological function in mammals. Glycobiology 13, 41R-53R (2003)

24. E.G. Zoetendal et al. The host genotype affects the bacterial community in the human gastrointestinal tract. Microb. Ecol. Health Dis. 13, 129-134 (2001)

25. R.E. Ley, F. Bäckhed, P. Turnbaugh, C.A. Lozupone, R.D. Knight, and J.I Gordon. Obesity alters gut microbial ecology. Proc. Natl. Acad. Sci. USA 102, 11070-11075 (2005)

26. J.F. Rawls, B.S. Samuel, and J.I. Gordon. Gnotobiotic zebrafish reveal evolutionarily conserved responses to the gut microbiota. 101, 4596-601.(2004) 
27. M. B. Roberfroid: Prebiotics and synbiotics: concepts and nutritional properties. Br J Nutr 80: S197-202 (1998)

28. G. E. Gibson, E. Tiernan, C. C. Cronin and J. B. Ferriss: Reversible bilateral ureteric obstruction due to a pancreatic pseudocyst. Gut 34, 1267-1268 (1993)

29. F.P. Martin, Y. Wang, N. Sprenger, I.K. Yap, T. Lundstedt, P. Lek, S. Rezzi, Z. Ramadan, P. van Bladeren, L.B. Fay, S. Kochhar, J.C. Lindon, E. Holmes, and J.K. Nicholson. Probiotic modulation of symbiotic gut microbial-host metabolic interactions in a humanized microbiome mouse model. Mol Syst Biol. 4:157 (2008)

30. A.A.de Graaf, and K. Venema. Gaining insight into microbial physiology in the large intestine: a special role for stable isotopes. Adv Microb Physiol. 53, 73-168 (2008)

31. P. Louis, K.P. Scott, S.H. Duncan and H.J. Flint. Understanding the effects of diet on bacterial metabolism in the large intestine. J. Appl. Microbiol. 102, 1197-1208.

32. L.C. Hoskins. Mucin degradation in the human gastrointestinal tract and its significance to enteric microbial ecology. Eur. J. Gastroenterol. Hepatol. 5, 203-213 (1992)

33. S. R. Cummings: Evaluating the benefits and risks of postmenopausal hormone therapy. Am J Med 91(5B): 14S-18S (1991)

34. L. Dethlefsen, P. B. Eckburg, E. M. Bik and D. A. Relman: Assembly of the human intestinal microbiota. Trends Ecol Evol 21, 517-23 (2006)

35. H. J. Flint: Polysaccharide breakdown by anaerobic microorganisms inhabiting the Mammalian gut. Adv Appl Microbiol 56, $89-120(2004)$

36. H. Brussow \& E Kutter. Phage ecology, In Bacteiophages: Biology and Applications (Kutter, E. and Sulakvelidze, A. eds), pp. 129-163, CRCPress (2005)

37. C. von Mering, P. Hugenholtz, J. Raes, S.G. Tringe, T. Doerks, L.J. Jensen, N. Ward \& Bork P. Quantitative phylogenetic assessment of microbial communities in diverse environments. Science. 315:1126-30 (2007)

38. P. Lepage, J. Colombet, P. Marteau, T. Sime-Ngando, J. Doré, and M. Leclerc Dysbiosis in inflammatory bowel disease: a role for bacteriophages? Gut. 57, 424-425 (2008)

39. C.F. Favier, E.E. Vaughan, W.M. De Vos, and A.D. Akkermans. Molecular monitoring of succession of bacterial communities in human neonates. Appl. Environ. Microbiol. 68, 219-26 (2002)

40. R. Mändar and M. Mikelsaar: Transmission of mother's microflora to the newborn at birth. Biol Neonate 69, 30-5 (1996)

41. A. Schwiertz, B. Gruhl, M. Löbnitz, P. Michel, M. Radke and M. Blaut: Development of the intestinal bacterial composition in hospitalized preterm infants in comparison with breast-fed, full-term infants. Pediatr Res 54: 393-9 (2003)

42. M. R. Millar, C. J. Linton, A. Cade, D. Glancy, M. Hall and H. Jalal: Application of 16S rRNA gene PCR to study bowel flora of preterm infants with and without necrotizing enterocolitis. J Clin Microbiol 34: 2506-2510 (1996)

43. A. Belenguer, S. H. Duncan, A. G. Calder, G. Holtrop, P. Louis, G. E. Lobley and H. J. Flint: Two routes of metabolic cross-feeding between Bifidobacterium adolescentis and butyrate-producing anaerobes from the human gut. Appl Environ Microbiol 72: 3593-3599 (2006)

44. H. S. M. Hayashi and Y. Benno: Phylogenetic analysis of the human gut microbiota using 16S rDNA clone libraries and strictly anaerobic culture-based methods. Microbiol Immunol 46, 535-548 (2002)

45. X. Wang, S. P. Heazlewood, D. O. Krause and T. H. Florin: Molecular characterization of the microbial species that colonize human ileal and colonic mucosa by using 16S rDNA sequence analysis. J Appl Microbiol 95, 508-520 (2003)

46. M.D. Collins, P.A. Lawson, A. Willems, J.J. Cordoba, J. Fernandez Garayzabal, P. Garcia, J. Cai, H. Hippe and J.A. Farrow. The phylogeny of the genus Clostridium: proposal of five new genera and eleven new species combinations. Int. J. Syst. Bacteriol. 44, 812-826 (1994) 
47. C. Manichanh, L. Rigottier-Gois, E. Bonnaud, K. Gloux, E. Pelletier, L. Frangeul, R. Nalin, C. Jarrin, P. Chardon, P. Marteau, J. Roca, and J. Dore. Reduced diversity of faecal microbiota in Crohn's disease revealed by a metagenomic approach. Gut. 55, 205-211. (2006)

48. C.C. Booijink, E.G. Zoetendal, M. Kleerebezem, and W.M. de Vos. Microbial communities in the human small intestine: coupling diversity to metagenomics. Future Microbiology. 2, 285-295 (2007)

49. K. Kurokawa, T. Itoh, T. Kuwahara, K. Oshima, H. Toh, A. Toyoda, H. Takami, H. Morita, V.K. Sharma, T.P. Srivastava, T.D. Taylor, H. Noguchi, H. Mori, Y. Ogura, D.S. Ehrlich, K. Itoh, T. Takagi, Y. Sakaki, T. Hayashi, and M. Hattori. Comparative metagenomics revealed commonly enriched gene sets in human gut microbiomes. DNA Res. 14, 169-181. (2007)

50. J. Xu: Microbial ecology in the age of genomics and metagenomics: concepts, tools, and recent advances. Mol Ecol 15, 1713$1731(2006)$

51. E. Furrie: A molecular revolution in the study of intestinal microflora. Gut 55, 141-143 (2006)

52. H. Hayashi, M. Sakamoto, M. Kitahara, and Y. Benno. Molecular analysis of fecal microbiota in elderly individuals using 16S rDNA library and T-RFLP. Microbiol Immunol. 47, 557-70 (2003)

53. E.G. Zoetendal, K. Ben-Amor, A.D. Akkermans, T. Abee, and W.M. de Vos. DNA isolation protocols affect the detection limit of PCR approaches of bacteria in samples from the human gastrointestinal tract. Syst Appl Microbiol. 24, 405-10 (2001)

54. E. G. Zoetendal, A. von Wright, T. Vilpponen-Salmela, K. Ben-Amor, A. D. Akkermans and W. M. de Vos: Mucosaassociated bacteria in the human gastrointestinal tract are uniformly distributed along the colon and differ from the community recovered from feces. Appl Environ Microbiol 68, 3401-3407 (2002)

55. E. G. Zoetendal, E. E. Vaughan and W. M. de Vos: A microbial world within us. Mol Microbiol 59, 1639-1650 (2006)

56. J. Raes, K.U. Foerstner, \& P. Bork. Get the most out of your metagenome: computational analysis of environmental sequence data. Curr Opin Microbiol. 10:490-8 (2007)

57. K. Gloux, M. Leclerc, H. Iliozer, R. L'Haridon, C. Manichanh, G. Corthier, R. Nalin, H.M. Blottière, and J. Doré. Development of high-throughput phenotyping of metagenomic clones from the human gut microbiome for modulation of eukaryotic cell growth. Appl Environ Microbiol. 73, 3734-3737 (2007)

Key Words: Intestinal microbiota, metagenomics, novel probiotic, probiotic bacteria

Send correspondence to: Dr. Marco Ventura, Department of Genetics, Biology of Microorganisms, Anthropology and Evolution University of Parma, Italy, Parco Area delle Scienze 11a, 43100 Parma, Italy, Tel: 339-521-905666, Fax: 339-521905604, E-mail: marco.ventura@unipr.it

Table1. Advantages and disadvantages of methods to analyze microbial communities.

\begin{tabular}{|c|c|c|}
\hline Method & Advantages & Disadvantages \\
\hline \multicolumn{3}{|l|}{ Traditional methods } \\
\hline Culture & $\begin{array}{l}\text { Provides isolates for further characterization; can recover } \\
\text { strains with desired traits; } \\
\text { physiological and biochemical studies are possible; } \\
\text { relatively inexpensive. }\end{array}$ & $\begin{array}{l}\text { Slow, time consuming and labour intensive; samples often require } \\
\text { immediate processing; restricted to culturable organisms and data } \\
\text { correspond to incomplete community representation; once isolated, } \\
\text { bacteria require identification using a number of techniques. }\end{array}$ \\
\hline \multicolumn{3}{|l|}{ 16S rRNA methods } \\
\hline $\begin{array}{l}\text { Oligonucleotide } \\
\text { hybridization (FISH, flow } \\
\text { cytometry, microarrays) }\end{array}$ & $\begin{array}{l}\text { Can be high throughput; can be very sensitive to very low } \\
\text { level of cells. }\end{array}$ & $\begin{array}{l}\text { Detects only taxa that hybridize to chosen probes, typically 6-18 genus } \\
\text { or family level group detected. }\end{array}$ \\
\hline $\begin{array}{l}\text { Community profiling } \\
\text { (real-time PCR) }\end{array}$ & Rapid, quantitative. & $\begin{array}{l}\text { Broad-range PCR bias; additional work to identify groups represented } \\
\text { in profiles; hard to compare analyses done at different times. }\end{array}$ \\
\hline 16S rRNA sequencing & $\begin{array}{l}\text { Identification to strain level; can discover novel taxa; can } \\
\text { analysis at multiple phylogenetic levels. }\end{array}$ & $\begin{array}{l}\text { Broad-range PCR bias; DNA extraction bias; expensive; laborious data } \\
\text { analysis. }\end{array}$ \\
\hline
\end{tabular}

Running title: Microbial diversity in the human intestine and novel insights from metagenomics

Figure 1. Schematic representation of metagenomics culture-independent approach. 


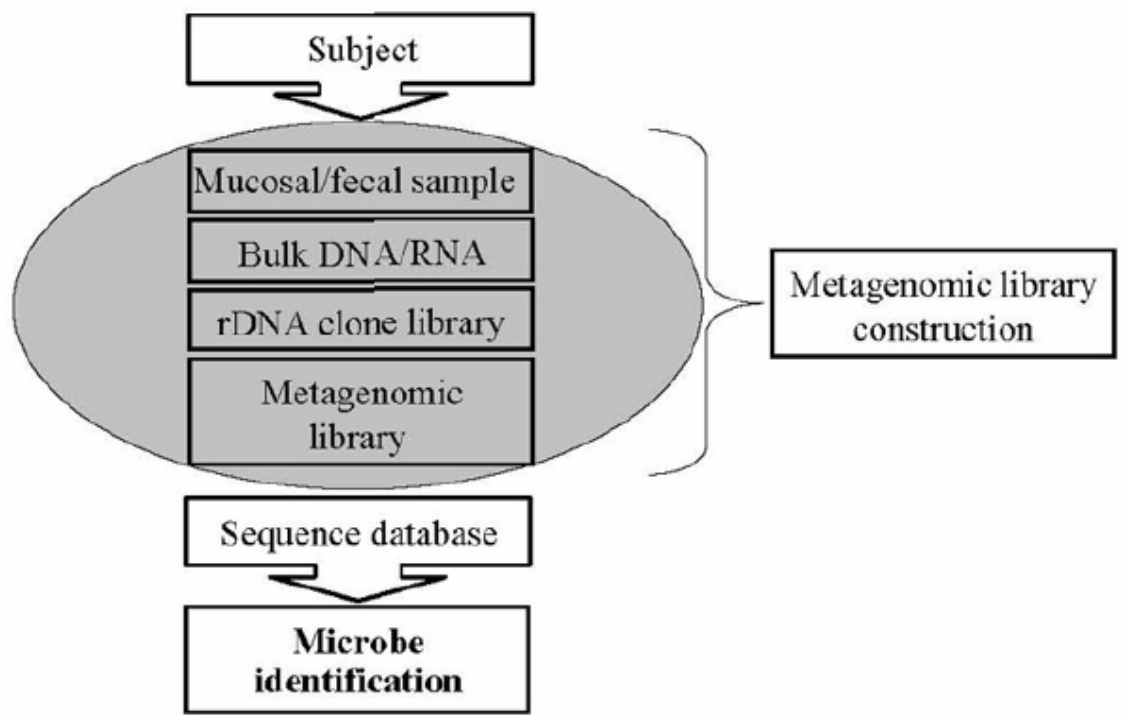

Figure 1. Schematic representation of metagenomics culture-independent approach. 